\title{
USO DE LA ORCIPRENALINA EN ALGUNOS CASOS OBSTETRICOS
}

\author{
Dr. Mauro Ortiz Pabón* \\ Dr. Donaldo de León V.**
}

\begin{abstract}
Departamento de Ginecología y Obstetricia - Hospital San Jorge - Pereira-Colombia. Trabajo presentado al IX Congreso Colombiano de Obstetricia y Ginecología. Bucaramanga, 5 al 11 de Diciembre de 1971.
\end{abstract}

\section{Introducción}

Este trabajo, que en gran parte ratifica publicaciones anteriores, lo hemos logrado pensando con criterio práctico, orientándonos exclusivamente por la clínica y con la esperanza de que pueda utilizarse en cualquier medio nuestro, con mínimos recursos para practicar la obstetricia.

Hace algún tiempo se viene experimentando con drogas presumiblemente inhibidoras de la contracción uterina, pero con resultados no muy satisfactorios $y$ en ocasiones, totalmente contradictorias (1). Solo en los últimos años se ha utilizado la Orciprenalina***, en ensayos preliminares $y$ muy especialmente en el Servicio de Fisiología Obstétrica de Montevideo.

El útero humano, posee receptores adrenérgicos alfa y beta; el estímulo de los receptores alfa produce efecto contráctil y la estimulación de los receptores adrenérgicos beta provoca efecto relajante de la contractilidad uterina (2). La Orciprenalina actúa deprimiendo la contracción uterina, casi exclusivamente a través del estímulo de los receptores beta adrenérgicos, disminuye el tono uterino, la intensidad de las contracciones y su frecuencia (3-4).
Otros efectos producidos por el mismo estímulo son: la broncodilatación, la inhibición de la motilidad intestinal y ureteral (2).

\section{Material y Método}

Como el sufrimiento fetal, de diferente etiología, termina con frecuencia en muerte del producto (5), hemos querido observar la acción de la Orciprenalina en algunos casos obstétricos.

Se estudiaron 14 casos, sin selección de edad, paridad, ni condición social, divididos así: 5 con amenaza de parto prematuro, cuya edad de embarazo estaba entre las 30 y 34 semanas; 3 casos de placenta previa entre el 40 y el $80 \%$ y 6 con inminencia de ruptura uterina.

Utilizamos la Orciprenalina, disolviendo 10 amps. de $0,5 \mathrm{mg}$. en 500 cc. de dextrosa en agua y a un goteo que oscilaba entre 10 y 20 gotas por minuto, con equipo de venoclisis de 10 gotas por cc., es decir, que ad-

* Ginecólogo Obstetra. Hospital Universitario del Valle (Cali).

** Ginecólogo Obstetra. Hospital Universitario de Caldas (Manizales).

** Alupent - C. H. Boehringer Sohm, Ingelheim am Rhein, Alemania. 
ministramos de 10 a 20 microgramos de orciprenalina por cc. Cuando fue necesario continuar por un tiempo más o menos largo con la droga, lo hicimos con tbs. de 20 mg., administrando una cada 8 horas.

Los resultados los valoramos clínicamente a los 15,30 y 60 minutos, sobre los siguientes parámetros:

En la Madre: Presión arterial y pulso.

En el feto: Frecuencia cardíaca e índice de Apgar tomado al nacer y a los 5 minutos.

\section{Resultados}

En los casos de amenaza de parto prematuro e inminencia de ruptura uterina, se obtuvo descenso muy apreciable de la actividad del útero entre los 15 y 30 primeros minutos y desaparición casi absoluta hacia los 60 minutos, efecto que también se reflejó en la reducción paulatina del sufrimiento fetal. En las pacientes con diagnóstico presuntivo de placenta previa, que más tarde se confirmó, el resultado lo observamos por disminución del sangrado vaginal y del sufrimiento fetal, como lo demuestran los Cuadros 1, 2 y 3.

En los tres grupos estudiados, la taquicardia materna se presentó en la casi totalidad de las pacientes hacia los 15 minutos, se mantuvo en general hacia los 30 y se normalizó a los 60 minutos.

La presión arterial materna, casi podríamos afirmar que no se modifica, pues hacia los 15 minutos el $50 \%$ permanecía inalterable, el $36 \%$ bajó y el $14 \%$ subió, pero estas variaciones no se salieron de las cifras normales y hacia los 30 minutos la totalidad de las madres tenían las presiones iniciales.
La fetocardia, que en 11 de nuestros casos se presentó con taquicardia y en 3 con bradicardia, aumentó su frecuencia en todos los casos hacia los 15 minutos, manteniéndose o elevándose más a los 30 minutos, para normalizarse más o menos a la hora de iniciado el goteo.

En cuanto al índice de Apgar: 9 fetos nacieron con valores de 70 más $y$ cinco fetos con puntaje de 60 menos, que a los cinco minutos y con maniobras de resucitación ya eran normales. Dos de estos últimos tenían al nacer circular de cordón apretado al cuello. Sólo un feto murió a las 12 horas de nacido, precisamente el producto de madre previamente hipertensa por nefropatía.

Vale la pena destacar que a una paciente con amenaza de parto prematuro y ruptura de membranas hacia las 34 semanas, fué llevada hasta las 38 semanas de gestación y parto normal con reposo, Orciprenalina y tratamiento antibiótico profiláctico. Otro caso que nos llamó la atención fué el de una paciente con diagnóstico presuntivo de placenta previa, a quien por error se le suspendió bruscamente la Orciprenalina y casi en seguida inició una fuerte hemorragia vía vaginal que obligó a la cesárea de inmediato.

\section{Resumen}

Ya en algunos centros científicos, especialmente en Montevideo, ha sido demostrado el potente poder inhibidor de la Orciprenalina sobre la contractilidad uterina. Nosotros abocamos el estudio de 14 pacientes con amenaza de parto prematuro, placenta previa e inminencia de ruptura uterina, todos ellos con sufrimiento fetal. Por tanto, nuestro trabajo tan solo pretende destacar la importancia de uso de esta droga en el sufri- 


\section{CUADRO NN 1}

VARIACION DE SIgNOS Vitales EN CINCO CASOS DE PARTO PREMATURO

\begin{tabular}{|c|c|c|c|c|c|c|c|c|c|c|c|}
\hline Signos Vitales & $\mathrm{N}^{0}$ & Alt. & $15 \operatorname{minutos}_{\text {Aum. }}$ & Dism. & $\mathrm{N}^{0}$ & Alt. & $\begin{array}{c}30 \text { minutos } \\
\text { Aum. }\end{array}$ & Dism. & Normal & $\begin{array}{l}60 \text { minutos } \\
\text { Aum. }\end{array}$ & Dism. \\
\hline Fetocardia & & - & 5 & - & & - & 5 & - & 5 & - & - \\
\hline Pulso & & 1 & 3 & 1 & & - & 5 & - & 5 & - & - \\
\hline Presión Arterial & & 1 & 2 & 2 & & 5 & - & - & 5 & - & - \\
\hline
\end{tabular}

CUADRO № 2

VARIACION DE SIGNOS VITALES EN TRES CASOS DE PLACENTA PREVIA

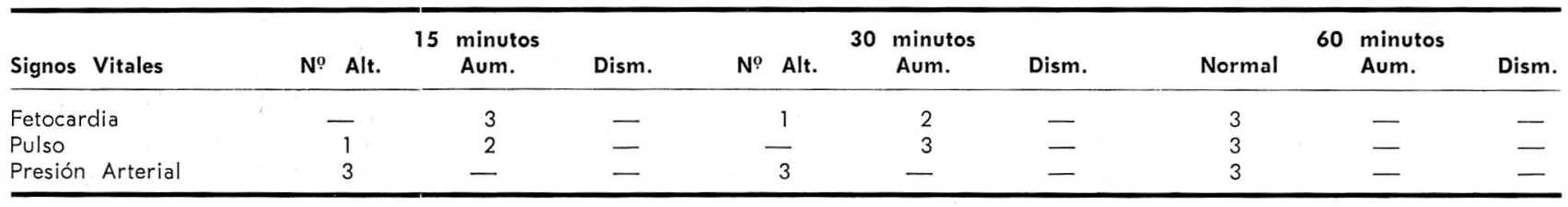

CUADRO № 3

VARIACION DE SIGNOS VITALES EN SEIS CASOS DE INMINENCIA DE RUPTURA UTERINA

\begin{tabular}{|c|c|c|c|c|c|c|c|c|c|c|c|}
\hline Signos Vitales & $\mathrm{N}^{0}$ & Alt. & $\begin{array}{l}15 \text { minutos } \\
\text { Aum. }\end{array}$ & Dism. & $\mathrm{N}^{0}$ & Alt. & $\begin{array}{l}30 \text { minutos } \\
\text { Aum. }\end{array}$ & Dism. & Normal & $\begin{array}{l}60 \text { minutos } \\
\text { Aum. }\end{array}$ & Dism. \\
\hline Fetocardia & & - & 6 & - & & - & 3 & 3 & 6 & - & - \\
\hline Pulso & & - & 6 & - & & - & 6 & - & 6 & - & - \\
\hline Presión Arterial & & 3 & - & 3 & & 6 & - & - & 6 & - & - \\
\hline
\end{tabular}


miento fetal de diversa etiología, demostrar la facilidad y sencillez de su manejo en el medio nuestro, comprobar el poco valor y frecuencia de efectos no deseables y llamar la atención sobre la curiosidad que despierta para posibles nuevas conquistas en la práctica obstétrica.

La taquicardia que apareció en todas las pacientes, hacia los 60 minutos ya tenía cifras normales. La presión arterial materna, en general, se mantuvo sin variaciones. El sufrimiento fetal disminuyó considerablemente, hasta el punto de obtener fetos con Apgar normal o que a los cinco minutos de nacidos se habían recuperado totalmente.

\section{Summary}

In certain scientific centers, particularly in Montevideo, the powerful inhibiting force of Orciprenalina on uterin contractability has already been demonstrated. We undertook the study of 14 patients with risks of premature birth, previous placenta and imminence of uterine rupture, all with fetal danger. Therefore, our work is merely intended to point the importance of the use of this medicine in fetal suffering of different ethiology, porve the advantage and simplicity of its use in our environment, prove the lettle value and frequency of undesirable effects and call the attentio nto the curiosity that arises for potential new findings in the obstetrical practice.

Tachycardia which appeared in all the patients, was again at normal figures towards the 60 minute count. The maternal arterial pressure remained un-changed. The fetal suffering considerably decreased to the point of having fetus with normal Apgar or which 5 minutes after birth had fully recovered.

\section{Conclusiones}

1. La potente acción útero-inhibidora de la Orciprenalina (4), la coloca como droga de elección, en todos aquellos casos obstétricos en que sea necesaria la reducción de la actividad uterina.

2. El sufrimiento fetal se beneficia particularmente (6), y en estos casos es posible obtener fetos normales o que se recuperan rápidamente.

3. Las pequeñas variantes de pulso y presión arterial maternas, así como la aparición ocasional de vómito, opresión precordial etc., carecen de importancia, pues en ningún momento son graves o irreversibles (2).

4. En casos de embarazo menor a las 38 semanas y probable placenta previa, el goteo de orciprenalina disminuye considerablemente el sangrado, con lo que se favorece la continuación del embarazo.

5. Aconsejamos el uso de la Orciprenalina, disolviendo 10 ampollas en $500 \mathrm{cc}$. de dextrosa al 5\% en agua y a un goteo que oscile entre 10 y 20 gotas por minuto, según la evolución del caso (4). Cuando la actividad uterina es muy alta inicialmente, pueden administrarse 2 ampollas de Orciprenalina directamente en la vena, antes de iniciar el goteo.

6. Si la paciente va a ser sometida a operación cesárea, es prudente suspender el goteo con alguna anticipación, pues puede presentarse hipotonía uterina después de la extracción del feto, complicación que por otra parte, cede rápidamente con masajes y ocitócicos intravenosos.

7. Presumimos el efecto positivo de la droga cuando se desee evitar la iniciación del trabajo de parto, acción que abriría un campo interesante en la práctica obstétrica. 
Agradecemos a los Laboratorios Boehringer Ingelheim, su decisiva colaboración para llevar a término este trabajo.

\section{BIBLIOGRAFIA}

1 BRUGGER A. "Contribución experimental al estudio de la espasmolitis uterina". Rev. Esp. de Gin. y Obst. 26: 137, 1967.

2 MAgAÑA J. M., GueVARA RUBIO G., POSEIRO J. J. Y CALDEIRO BARCIA R. "Acción de la Orciprenalina sobre la contractilidad del útero humano grávido, el sistema cardiovascular materno y la frecuencia cardíaca fetal". Memorias. Montevideo, 1969. 1 : $615-630$

3 COBO E., KAFURY S. "Inhibición de la Contractilidad del útero en la amenaza de parto prematuro mediante el uso de la Orciprenalina". Rev. Col. de Obst. y Gin. Vol. 21: 111-117, 1970.

4 SOTO YANCES A. "Uso clínico de la Orciprenalina como inhibidor de la Contractilidad uterina". Impreso. Facultad de Medicina U. de Cartagena.

5 ESCOBAR SOTO J., GOMEZ RUEDA A. "Mortalidad perinatal". Rev. Col. de Obst. y Gin. 15: 295, 1964.

6 CALDEIRO BARCIA R., MAGAÑA J. M. Y col. "Nuevo enfoque para el tratamiento del sufrimiento fetal agudo intraparto". Archivos Uruguayos de Gin. y Obst. 24: 1-2, IV-VIII-69. 